\title{
Psychological and educational interventions for preventing falls in older people living in the community (Protocol)
}

Udell JE, Drahota A, Mackenzie H

Udell JE, Drahota A, Mackenzie H.

Psychological and educational interventions for preventing falls in older people living in the community.

Cochrane Database of Systematic Reviews 2019, Issue 11. Art. No.: CD013480.

DOI: $10.1002 / 14651858 . C D 013480$.

www.cochranelibrary.com 
TABLE OF CONTENTS

HEADER 1

ABSTRACT

BACKGROUND

OBJECTIVES

METHODS

ACKNOWLEDGEMENTS

REFERENCES

APPENDICES

CONTRIBUTIONS OF AUTHORS

DECLARATIONS OF INTEREST

SOURCES OF SUPPORT 
[Intervention Protocol]

\section{Psychological and educational interventions for preventing falls in older people living in the community}

Julie E Udell ${ }^{1}$, Amy Drahota ${ }^{2}$, Heather Mackenzie²

1Department of Psychology, University of Portsmouth, Portsmouth, UK. 2School of Health and Care Professions, University of Portsmouth, Portsmouth, UK

Contact address: Julie E Udell, Department of Psychology, University of Portsmouth, King Henry Building, King Henry 1 St, Portsmouth, Hampshire, PO1 2DY, UK. julie.udell@port.ac.uk.

Editorial group: Cochrane Bone, Joint and Muscle Trauma Group Publication status and date: New, published in Issue 11, 2019.

Citation: Udell JE, Drahota A, Mackenzie H. Psychological and educational interventions for preventing falls in older people living in the community. Cochrane Database of Systematic Reviews 2019, Issue 11. Art. No.: CD013480. DOI: 10.1002/14651858.CD013480.

Copyright (c) 2019 The Cochrane Collaboration. Published by John Wiley \& Sons, Ltd.

\section{A B S T R A C T}

This is a protocol for a Cochrane Review (Intervention). The objectives are as follows:

- To assess the effects (benefits and harms) of psychological interventions (such as cognitive behavioural therapy) (with or without an education component) for preventing falls in older people living in the community.

- To assess the effects (benefits and harms) of educational interventions for preventing falls in older people living in the community. 


\section{B A C K G R O U N D}

\section{Description of the condition}

A consensus definition of a fall is "an unexpected event in which the participant comes to rest on the ground, floor, or lower level" (Lamb 2005). This definition, informed by a systematic review (Hauer 2006), has been incorporated by the Prevention of Falls Network Europe (ProFaNE) group into their development of common outcome definitions and measurements for falls prevention research (Lamb 2005). A fall is unlikely to be attributable to a single cause. A complex combination of intrinsic factors (to do with the person), extrinsic factors (external to the person) or situational factors (or a combination of these three) can lead to a fall. The actual combination of factors is very much related to each individual's circumstances and health status (Healey 2007; Speechley 2011).

It is thought that intrinsic risk factors are most likely for people aged 80 and over because loss of consciousness is more common in this age group, while extrinsic risk factors are most likely to be responsible for falls in people younger than 75 years old (Todd 2004). Intrinsic fall risk factors may be related to the individual's age and include changes in vision, balance, the musculoskeletal and cardiovascular systems. For example, changes in vision may reduce the individual's ability to assess the environment for potential hazards, especially when there is a reduction in visual clarity, the speed at which the eyes can adjust to differences in dark and light, or contrast sensitivity. Floor surfaces may appear dipped or raised if the person suffers a decrease in depth perception, while a decreased sensitivity to glare caused by light means that a person might have more difficulty in perceiving the slipperiness of a floor surface. This can lead to an altered walking pattern to compensate for the perceived risk, which can result in instability (Tideiksaar 2002).

Extrinsic fall risk factors occur in the physical environment, externally to the person, and may include objects such as loose rugs, lighting, footwear, or even medication. These falls are usually due to either an unfortunate encounter with a known environmental hazard or due to the person's increased risk in relation to hazards because of their age, health problems or both of these (Healey 2007; Rubenstein 2006). Health professionals, such as occupational therapists, have a major role in the implementation of environmental hazard reduction (Gill 2000); they can accomplish this by visiting the patient's home and giving them advice on managing environmental hazards that may increase the possibility of a fall. Extrinsic risks can also be connected with the person's medication. For example, a single medication may cause side-effects that can increase the person's risk of falling. However, when several medications are prescribed at the same time (polypharmacy), there is the possibility of an interaction between them, depending upon the person (Tinetti 2010). Situational factors relate to the circumstances of a person's fall risk; for example, the time of day (many falls occur at night).

A physical injury from a fall can range from superficial cuts and bruises, through to more severe injuries such as a hip fracture (Lord 2007). After a hip fracture, many people will sustain ongoing limitations that can have a major impact on their quality of life. As well as physical consequences, falls can also have psychological consequences such as anxiety, depression, and loss of self-esteem; and also fear of falling, which can result in perceived poor health, avoidance of activity, worry about loss of independence, and loss of confidence (CDHAC 2001; Cumming 1999; Yardley 2002; Zijlstra 2007).
Once an individual has fallen, the fear of falling again may act as a risk factor for future falls (Speechley 2011). Additionally, many older people view a fall as a sign of ageing, indicating a reduction in competence, and an increase in dependence (Aminzadeh 1998). Falls are not therefore a purely 'physical' health problem, but rather can be seen to both influence, and be influenced by, cognitive and mental constructs.

Approximately one in every three older adults is estimated to fall at least once each year (WHO 2007). Reported prevalence rates do, however, vary widely from region to region: for example, the prevalence of falls in older adults living in the Gulf Cooperation Council countries is estimated to be $46.9 \%$ (Alqahtani 2019), whilst in Canada it is estimated to be $20.1 \%$ (Chang 2015). Each year globally there are 37.3 million falls which are considered severe enough to require medical attention (WHO 2018a). Resulting from these falls 646,000 people, most of whom will be aged 65 years or older, die from related injuries (WHO 2018a). Each fall-related injury has an economic impact for the health care system of each country. For example, the WHO 2018a suggests that for Finland and Australia the average cost per fall injury can be USD 3611 and USD 1049 respectively. In the UK, NICE 2013 estimated that the annual total costs to the NHS alone from falls among older people is GBP 2.3 billion. These human and economic costs have a huge impact on health care systems.

\section{Description of the intervention}

Many of the risk factors for falls can be reversed or corrected. This review will cover two key categories of interventions, psychological and educational, that are part of the array of interventions used to reduce the risk of falls. In this review, we will align the definitions of these two categories with those (respectively 'Psychological' and 'Knowledge') in the standardised fall prevention interventions taxonomy established by the ProFaNE project (Lamb 2011).

A psychological intervention is a treatment that works to modify thoughts, feelings, behaviour or emotional state (O'Shea 2017); with regards to fall prevention, it may be used with the aim of identifying and then adapting thought processes that may increase falls, and also to encourage the individual to change their behaviour concerning falling and fall prevention (Clemson 2004). The interventions may be conducted with individuals or within a group setting (Dryden 2012). One of the most commonly known psychological therapies is Cognitive Behavioural Therapy (CBT). Although the roots of CBT can be traced back to philosophers such as the Buddha and Epictetus, the term 'Cognitive Behavioural Therapy' was only adopted in the 1970s (Mansell 2012). The ProFaNE Taxonomy (ProFaNE 2007) adopts the National Center for Biotechnology Information Medical Subject Headings (NCBI MeSH) definition for CBT as follows: "A direct form of psychotherapy based on the interpretation of situation (cognitive structure of experiences) that determine how an individual feels and behaves. It is based on the premise that cognition, the process of acquiring knowledge and forming beliefs, is a primary determinant of mood and behaviour. The therapy uses behavioural and verbal techniques to identify and correct negative thinking that is at the root of the aberrant behaviour" (MeSH D015928). A less technical definition of CBT from NHS Guidance (UK) is: "Cognitive behavioural therapy (CBT) is a talking therapy that can help you manage your problems by changing the way you think and behave" (NHS 2019). Psychological interventions may also include an element of education; for example, increasing knowledge on types of fall risks. 
Educational interventions cover any form of education - including videos, lectures, and leaflets - which provides the receiver with knowledge about falls prevention. More recently, education concerning falls prevention is being delivered through the use of emerging technologies, for example virtual reality; Le 2015 provides an example from the construction industry. Virtual reality or 'VR' is a "computer-generated simulation of a three-dimensional image or environment that can be interacted with in a seemingly real or physical way by a person using special electronic equipment, such as a helmet with a screen inside or gloves fitted with sensors" (Lexico 2019). Additionally, researchers have explored the acceptability and usability of the internet to provide guidance about exercise for falls prevention and, although the advice was well received by the participants, it was recognised that only a minimal number of older people accessed the internet at that time (Nyman 2009; Yardley 2007). This position may be changing, however, as more older adults are engaging with the internet (Duggan 2015; Frith 2017). As well as being internet-based, interventions may be delivered face-to-face or via hard-copy media (e.g. printed leaflets or booklets). The content of the intervention may be personalised or generic, and cover a range of topics, such as identifying risk factors that may be intrinsic (e.g. balance, strength, medications) or extrinsic (e.g. home hazards, such as loose rugs), and how to reduce risk. The success of these forms of passing on information may depend upon the person's perspective of their own risk of falling, and if they do not think they are at risk of falling they may not be interested in the educational content. Indeed, even if they do engage with the information, they may still not have the resources to carry out the advice (Ballinger 2002).

\section{How the intervention might work}

Whether an injury is sustained or not, a fall may still bring about psychosocial issues such as anxiety, depression and loss of self-esteem and confidence. Fear of an initial or subsequent fall can result in perceived poor health, avoidance of activity, worry about loss of independence and loss of confidence (CDHAC 2001; Yardley 2002; Zijlstra 2007). This can lead to a restriction of physical activities which, ironically, increases the risk of falling (Reventlow 2007; Speechley 2011). Falls can also result in hospital admission and increase the likelihood of care home admission (Healey 2007). As Huang 2011 highlights, falling is not only affected by physical conditions but also by the psychological effects; the aim of these interventions is therefore to identify and then adapt thought processes that may increase falls (Clemson 2004). Evidence indicates, however, that this is an area that still needs further research (Cameron 2018; Gillespie 2012).

Psychological interventions, such as mindfulness or CBT, often go hand in hand with educational interventions, which seek to raise awareness of the factors which can contribute to falls, and therefore aim to encourage individuals to make positive changes to their lifestyles and environmental surroundings to reduce the risk of falls. Educational interventions may also help overcome the myth that falls are an inevitable part of ageing and cannot be prevented (Aminzadeh 1998). In this way, educational interventions may seek to change attitudes towards falls prevention, tackle the perception that the need to engage in falls prevention interventions is an undesired sign of ageing, increase knowledge about potential interventions, and encourage uptake and implementation of risk prevention strategies.
Nyman 2011 discusses how knowledge and attitudes may impact upon falls prevention behaviours within the context of the Theory of Planned Behaviour (Ajzen 1988). This theory suggests that behaviour changes come about through intentions, which are an indication of the level of effort a person is planning to put towards performing the behaviour. The person's intentions to carry out the behaviour can, however, be affected by their attitude towards the behaviour, the perceived social pressure to carry out the behaviour, and how much confidence they feel that they have in actually being able to carry out the behaviour (Ajzen 1988). Educational and psychological interventions may target these prerequisites for behaviour change; it has also been suggested, however, that changes to knowledge and attitudes may not be sufficient, in and of themselves, to lead to improved falls prevention practices (Nyman 2011). This systematic review will therefore explore the extent to which educational and psychological interventions are effective at preventing falls.

\section{Why it is important to do this review}

This review will update the evidence for two key sets of interventions of those covered in the all-intervention review of Gillespie 2012. Updates of the evidence for other intervention categories covered in Gillespie 2012 are either available (Hopewell 2018; Sherrington 2019), in preparation (Clemson 2019), or planned. The number of people over the age of 60 continues to rise. In 2015, there were 900 million people aged 60 years and older globally, and this is predicted to rise to 2 billion by 2050 (WHO 2018). This, along with a growing number of studies focusing on psychological or educational interventions for falls prevention, means that it is important to summarise the effectiveness of the evidence in this field to inform decision-making for people aiming to reduce the number of falls for older adults in the community.

\section{O B JECT IVES}

- To assess the effects (benefits and harms) of psychological interventions (such as cognitive behavioural therapy) (with or without an education component) for preventing falls in older people living in the community.

- To assess the effects (benefits and harms) of educational interventions for preventing falls in older people living in the community.

\section{METHODS}

\section{Criteria for considering studies for this review}

\section{Types of studies}

We will include randomised controlled trials, randomised at either the individual or cluster level, evaluating the effects of psychological or educational interventions on the incidence of falls in older people living in the community. We will exclude trials that explicitly use methods of quasi-randomisation (e.g. allocation to groups by alternation or date of birth). We will include cluster randomised trials because these might be a more suitable design where there is a risk of contamination - for example, where educational materials are being distributed on a large scale. In this case, it is possible that control participants may become aware of, and adopt, the falls education provided to participants in the intervention condition. We do not consider cross-over trials to be suitable for this review question (where participants are first randomised to receive either intervention or control, and then 'cross over' into the other 
arm of the study), since the effects of the interventions we are interested in have carry-over effects (they cannot be 'undone' once they have been delivered). We will include studies irrespective of their language and publication status.

\section{Types of participants}

We will include trials if they specify an inclusion criterion of 60 years of age or over. We will include trials comprising younger participants if the mean age minus one standard deviation is more than 60 years. We propose to include trials where the majority of participants are living in the community; this includes people who are living in domestic accommodation or in places of residence that, on the whole, do not provide residential health-related care or rehabilitative services - for example, retirement villages or sheltered housing.

Trials with mixed populations (community and higher dependency places of residence) will be eligible for inclusion if data are provided for subgroups based on setting or the numbers in higher dependency residences are very few and balanced in the comparison groups.

We propose to include trials recruiting participants from hospitals or other institutions if the majority were discharged to the community (where the majority of the intervention was delivered and falls were recorded). We will exclude studies that test interventions for preventing falls in people affected by particular clinical conditions such as stroke, Parkinson's disease, multiple sclerosis and dementia. Several of these topic areas are covered by other Cochrane Reviews (Canning 2015; Verheyden 2013). We acknowledge that some individuals with these (and other) health conditions may be included in studies of the general community but we will only exclude studies in which all participants have a particular condition.

\section{Types of interventions}

This review will include all psychological and educational interventions tested in trials that are targeting falls prevention in older people. We will consider these two groups of interventions separately as follows.

\section{Psychological interventions}

We will include all trials where a psychological intervention is delivered as a single intervention as opposed to a component of a broader intervention. We will include trials where an additional low-contact intervention was given to one or both groups if we judge that the main purpose of the study was to investigate the effect of psychological interventions. We will also include trials where the psychological intervention featured a strong educational component.

Psychological interventions will be examined and then categorised to one of the following categories.

- Cognitive Behavioural Therapy (CBT) interventions

- Dialectical Behavioural Therapy (DBT) interventions

- Mindfulness interventions

- Other psychological interventions not listed above (e.g. Acceptance and Commitment Therapy (ACT) etc.)

We will compare each of these types of psychological intervention with a control condition, comprising either 'usual care' (i.e. no change in usual activities) or another control intervention (i.e. an intervention that is not thought to reduce falls, such as general health education, social visits or very gentle exercise not expected to impact on falls). To clarify that we would also include trials where a similar education intervention was used in both arms of the trial: i.e. psychological intervention plus education versus education alone.

We recognise the very broad nature of these categories, and therefore plan to undertake a more in-depth assessment of the types of interventions provided in our exploration of heterogeneity.

\section{Educational interventions}

We will include all trials where an educational intervention is delivered as a single intervention as opposed to a component of a broader intervention (e.g. many exercise studies may incorporate a 'training' component to educate people on how to perform their exercises as prescribed), with the exception of where education forms part of a psychological intervention (as described above). The content of the education materials may cover single, multiple, or multifactorial interventions where the selection of educational intervention(s) is determined by an assessment of a person's falls risk. Examples of the topics that these educational interventions might cover include: identifying health conditions that increase falls risk, preventing falls in the home, benefits of exercise to prevent falls, and the role of nutrition in falls prevention. We will include trials where an additional low-contact intervention was given to one or both groups if we judge that the main purpose of the study was to investigate the effect of educational interventions. We will define the content of the educational intervention according to whether it pertains to: (1) a single intervention - one in which the participants receive education on only one topic relating directly to falls prevention (we will document the topic); (2) multiple component intervention - one in which interventions include two or more topics relating directly to falls prevention; and (3) multifactorial educational interventions - ones in which the educational interventions includes two or more topics directly relating to falls prevention but where the selection of these is linked to an assessment of the individual's falls risk profile. Thus, each individual receives a fall risk assessment and then receives an intervention to match their risk profile (e.g. one person may receive education about the benefits of doing exercise to improve muscle strength and education about how to modify falls risks at home, whereas another may receive education about how to modify falls risks at home and how to cope with urinary continence and the associated falls risks).

We will compare each of these types of educational interventions with a control condition, comprising either 'usual care' (i.e. no change in usual activities) or another control intervention (i.e. an intervention that is not thought to reduce falls, such as general health education, social visits or very gentle exercise not expected to impact on falls). We recognise the very broad nature of these categories, and therefore plan to undertake a more in-depth assessment of the types of interventions provided in our exploration of heterogeneity.

For both intervention categories, studies that utilise 'waiting list' controls will be considered eligible for inclusion, where outcomes are assessed at the end of the waiting list period.

\section{Types of outcome measures}

The reporting of specific outcomes does not form part of the eligibility criteria for our review; however the purpose of the interventions should be to reduce falls, or reduce falls secondary to psy- 
chological outcomes such as fear of falling. We have discussed our outcome measures with our public involvement group and sought to align them, where possible, with other Cochrane Reviews (e.g. Hopewell 2018) and the recommended outcome set for falls injury prevention studies in the community (Lamb 2005). Our public involvement members have informed the priority of our outcomes and the inclusion of additional outcomes around psychological well-being.

There are a number of scales used to measure falls-related psychological outcomes, to capture feelings of self-efficacy, anxiety, confidence, or fear of falling. These outcomes and the respective tools have come under criticism for being conflated with each other and misinterpreted (Adamczewska 2018). In order to deal with this situation, our approach will be to consider falls-related psychological outcomes on a scale-by-scale basis, so whilst we will present them together (sub-grouped by measurement tool), we will not combine them in a meta-analysis of different tool types.

We will consider economic outcome data where available, and will align our approach for the incorporation of costs data to an exemplar systematic review by Garrison 2010, and another review we are conducting (Drahota 2019).

\section{Primary outcomes}

- Rate of falls (number of falls; falls per person-year).

\section{Secondary outcomes}

- Number of fallers (i.e. number of people experiencing one or more falls; risk of falling).

- Number of people experiencing one of more fall-related fractures.

- Number of participants experiencing one or more falls that required medical attention.

- Number of people experiencing one or more falls that resulted in hospital admission.

- Fall-related psychological outcomes (measured using a validated scale, e.g. FES-I (Yardley 2005) (short-term: < 6 months; medium term: $\geq 6$ months to $<12$ months, and long-term follow-up: $\geq 12$ months).

- Health-related quality of life (measured using a validated scale, e.g. EQ-5D (e.g. Herdman 2011) or similar; with short-, medium-, and long-term follow-up as above).

- Number of participants experiencing one or more adverse events.

We will extract health economic data (cost utilisation, cost benefit, and cost effectiveness) where the information is available.

Where included studies report on outcomes that capture patient experiences or satisfaction with the interventions, we will summarise these in the review.

\section{Search methods for identification of studies}

\section{Electronic searches}

Our search will extend the searches performed up to February 2012 in Gillespie 2012. Using tailored search strategies we will search the Cochrane Bone, Joint and Muscle Trauma Group Specialised Register (February 2012 to present); the Cochrane Central Register of Controlled Trials (CENTRAL) (Cochrane Register of Studies
(CRS Web); 2012 Issue 3 to current issue) in the Cochrane Library; MEDLINE (via Ovid, March 2012 to present); Embase (January 2019 to present); CINAHL (Cumulative Index to Nursing and Allied Health Literature; February 2012 to present); PsycINFO (February 2012 to present); OT seeker (Occupational Therapy Systematic Evaluation of Evidence; to present); and NHS Economic Evaluation Database (NHS EED; inception to present).

We will not apply any language restrictions. In MEDLINE, we will combine subject-specific search terms with the sensitivity-and precision-maximising version of the Cochrane Highly Sensitive Search Strategy for identifying randomised trials (Lefebvre 2011). An example search strategy for MEDLINE (via Ovid) is shown in Appendix 1. This will be adapted for the other databases.

We will also search the World Health Organization International Clinical Trials Registry Platform (WHO ICTRP) and the US National Institutes of Health Ongoing Trials Register (ClinicalTrials.gov) for ongoing and recently completed trials.

\section{Searching other resources}

We will check reference lists of other systematic reviews and of the included studies. We will also contact researchers in the field to identify ongoing and unpublished trials. We will undertake forward citation searches of any included studies, using Google Scholar.

\section{Data collection and analysis}

\section{Selection of studies}

Two review authors will independently screen all titles and abstracts for studies which are potentially eligible. We will obtain full text for these studies and two review authors will independently assess eligibility for inclusion. We will resolve any disagreements by discussion or, if necessary, by inviting a third review author to arbitrate.

\section{Data extraction and management}

Two review authors will independently carry out the data extraction. We will develop and pilot a data collection form on two studies to ensure that all the required items and instructions are included in the form. The two authors will resolve disagreements through discussion; or if necessary they will consult with the third author. The review authors will not be blind to the names of authors, institutions, journals or outcomes. Our data collection form will record the following items.

- General information: review author's name, date of data extraction, study ID, first author, author's contact address/email (if available), citation of paper and trial objectives.

- Trial details: trial design, location, setting, recruitment period, sample size, inclusion and exclusion criteria, comparability of groups, length of follow-up, stratification, stopping rules, patient and public involvement, and funding source.

- 'Risk of bias' assessment: sequence generation, allocation concealment, blinding (participants, personnel, outcome assessors), incomplete outcome data, selective outcome reporting and other bias (recall bias).

- Characteristics of participants: age, gender, ethnicity, the number randomised, analysed, losses to follow-up and dropouts in each arm (with reasons).

Psychological and educational interventions for preventing falls in older people living in the community (Protocol) 
- Interventions: experimental and control interventions, duration, intensity, mode of delivery, personnel involved in delivering the intervention (professions, training), whether studies assessed adherence (compliance) with interventions, and additional co-interventions.

- Outcome data acquisition: how falls were identified and collected, how injuries were classified and measured, tools used for measuring patient-reported outcomes (scale used, direction of scale, range of feasible scores, minimally important difference if known), timings of outcome data acquisition.

- Outcomes measured: rate of falls, number of people sustaining one or more falls, number of people sustaining one or more fallrelated fractures, number of people who experience a fall requiring medical attention, number of people who experience a fall requiring hospital admission, falls-related psychological outcomes, health-related quality of life and adverse effects of the intervention.

For economic evaluations, our data collection form will be based on the format and guidelines used to produce structured abstracts of full economic evaluations for inclusion in the NHS Economic Evaluation Database. Data extraction will include study characteristics such as country, settings, aims, and methodological aspects related to economic evaluation, individual items within the respective checklists (Drummond 1996; Philips 2006), and the following economic variables, if reported: costs of intervention; costs of falls based on injury, such as healthcare resources (e.g. hospital admissions, outpatient visits, surgery needs); utility measures such as quality of life, life years and quality-adjusted life years; and summary measures such as incremental cost-effectiveness ratios (ICERs), net monetary benefits, and value of information (Vol). We will classify economic evaluations by type (Partial evaluations: 'outcome description', 'cost description', 'cost-outcome description', 'efficacy or effectiveness evaluation', or 'cost analysis'; Full economic evaluations: 'cost-effectiveness analysis', 'cost-utility analysis', or 'cost-benefit analysis') and as either an economic evaluation based on a single study or a model-based economic evaluation.

We will retrieve data from both full-text and abstract reports of studies. Where these sources do not provide sufficient information, we will contact study authors for additional data.

\section{Assessment of risk of bias in included studies}

Two review authors will independently assess the risk of bias of each included study using Cochrane's 'Risk of bias' tool as described in the Cochrane Handbook for Systematic Reviews of Interventions (Higgins 2017). Review authors will not be blind to author or source institution. The review authors will resolve any disagreements by discussion or, if they cannot achieve consensus, a third review author will act as arbitrator. As outlined in Appendix 2, we will assess the following domains: random sequence generation (selection bias); allocation concealment (selection bias); blinding of participants and personnel (performance bias); blinding of outcome assessment (detection bias, for each outcome separately); incomplete outcome data (attrition bias); and selective outcome reporting bias. We will also assess bias in the recall of falls due to less reliable methods of ascertainment (Hannan 2010). Regarding risk of bias, we will rate this as low, high or unclear for each domain.

We will assess the methodological quality of included economic evaluations through the use of recognised checklists based on guidelines for economic submissions to the British Medical Jour- nal (for economic evaluations based on a single study; Drummond 1996), and for quality assessment in economic decision-analytic models (for model-based economic evaluations; Philips 2006).

Our public involvement members will be involved in assessing how we report our risk of bias judgements to help ensure that we are clear, transparent, and consistent with how we are making our judgements.

\section{Measures of treatment effect}

We will report the treatment effect for rate of falls, rate of fall-related fractures, rate of falls that required medical attention, and rate of falls that resulted in hospital admission, as rate ratios (RaRs) with $95 \%$ confidence intervals (Cls). Where adjusted and unadjusted rates are presented, we will use the unadjusted figures, unless the adjustment is for clustering. For the number of fallers, number sustaining fall-related fractures, number experiencing falls that required medical attention, number of people experiencing one or more falls that resulted in hospital admission, and number of adverse events, we will report risk ratios (RRs) and $95 \% \mathrm{Cls}$. For continuous outcomes (e.g. health-related quality of life and falls-related psychological outcomes) we will present the mean difference (MD) with $95 \% \mathrm{Cls}$ where the same outcome measure is used, or standardised mean difference (SMD) with $95 \%$ Cls for outcomes measured using different scales.

\section{Unit of analysis issues}

In order to avoid unit-of-analysis issues (including the same group of participants more than once in an analysis), we will be mindful of trials with multiple associated publications, or with multiple intervention groups; in the case of multiple study arms, we will either combine the groups (if logical) or include only one pairwise comparison (intervention versus control) in any one analysis. If it makes logical sense to include multiple pairwise comparisons (intervention versus control) in the analyses, we will avoid including the same group of participants twice by 'splitting' the control group, distributing the number of control group participants to each analysis in proportion to the number of participants in each intervention group.

We will clearly identify cluster randomised trials in the review and describe the way that we have dealt with the data. Where clustering has not been taken into account, we plan to adjust the estimates using an intra-cluster correlation coefficient borrowed from another similar study. For this, we will align our approach to dealing with unit-of-analysis issues to that of other Cochrane Reviews on falls prevention (e.g. Hopewell 2018). For trials that are cluster randomised (e.g. by medical practice), we will perform adjustments for clustering as described in Higgins 2017 if this was not done in the published report. We will use an intraclass correlation coefficient (ICC) of 0.01 as reported in Smeeth 2002. We will ignore the possibility of a clustering effect in trials randomising by household.

We will be alert to the unit-of-analysis issues relating to outcome reporting at different follow-up times; and the presentation of outcomes, such as adverse events, by the number of outcomes rather than participants with these outcomes.

\section{Dealing with missing data}

We will attempt to contact the study investigators for any key missing or unclear data or information on their trial. We will aim to 
use open-ended questions in order to avoid influencing the direction of the responses. If missing data are from participant/cluster dropouts, we will conduct analyses based on the available data and include an assessment of the problem as part of our risk of bias judgements. If a study does not report SDs for continuous outcomes, we will calculate these from standard errors, $\mathrm{Cls}$ or exact probability $(P)$ values where possible.

\section{Assessment of heterogeneity}

The decision about whether or not to combine the results of individual studies will depend on an assessment of clinical and methodological heterogeneity. We will explore heterogeneity irrespective of whether we decide to pool studies in a meta-analysis. We will assess heterogeneity through a combination of visual inspection of the forest plots, along with consideration of tests for homogeneity $\left(\mathrm{Chi}^{2}\right.$ with statistical significance set at $\left.\mathrm{P}<0.10\right)$, and measures for inconsistency $\left(\mathrm{I}^{2}\right)$ and heterogeneity $\left(\mathrm{Tau}^{2}\right)$. We will base our interpretation of the $\mathrm{I}^{2}$ results on that suggested by Higgins 2017: $0 \%$ to $40 \%$ might not be important; $30 \%$ to $60 \%$ may represent moderate heterogeneity; $50 \%$ to $90 \%$ may represent substantial heterogeneity; and $75 \%$ to $100 \%$ may represent very substantial ('considerable') heterogeneity. Recognising that the use of simple thresholds to assess heterogeneity is problematic, particularly when dealing with few studies, we will also consider the magnitude and direction of the effects observed in the forest plots. We will also look holistically at the measures available to us $\left(\mathrm{Chi}^{2}, \mathrm{I}^{2}, \mathrm{Tau}^{2}\right)$ to take into account the strength of the evidence for heterogeneity, alongside the guide for interpreting $I^{2}$ values provided above.

\section{Assessment of reporting biases}

Where possible, we will draw funnel plots with different plotting symbols to identify subgroups (as specified below). We will only test for funnel plot asymmetry if there are sufficient data (at least 10 studies to be combined), and will use visual inspection of the plots to interpret the findings. Our aim is to reduce the risk of publication bias affecting our results by conducting a thorough search and communicating with researchers in the field.

\section{Data synthesis}

We will classify interventions according to whether they are psychological (with or without an education component), or education alone, and analyse these separately. Using the classification systems developed for the ProFaNe taxonomy (Lamb 2005), as proposed in this protocol, we will group similar psychological interventions for analysis; and then separately, we will group similar educational interventions for analysis.

Where appropriate, we will pool the results of comparable studies using both fixed-effect and random-effects models. The choice of the model to report will be guided by careful consideration of the extent of heterogeneity and whether it can be explained, in addition to other factors such as the number and size of included studies. We will use $95 \% \mathrm{Cls}$ throughout. We will consider not pooling data where there is considerable heterogeneity $\left(I^{2} \geq 75 \%\right)$ that cannot be explained by the diversity of methodological or clinical features among trials. Where pooling data is inappropriate, we will still present trial data in the figures and tables for illustrative purposes and will report these in the text.

When considered appropriate, we will pool data using the generic inverse variance method in Review Manager Web. This method en- ables pooling of the adjusted and unadjusted treatment effect estimates (rate ratios or risk ratios) reported in the individual studies or that can be calculated from data presented in the published article (see Measures of treatment effect). The generic inverse variance option in Review Manager Web requires entering the natural logarithm of the rate ratio or risk ratio and its standard error for each trial; we will calculate these in an Excel spreadsheet as required.

For economic data, we will tabulate and summarise the results narratively in the text. To facilitate comparisons between studies, we will adjust all costs to 2020 International Dollar values using a webbased conversion tool (Cost Converter) that has been developed by The Campbell and Cochrane Economics Methods Group (CCEMG) and the Evidence for Policy and Practice Information and Coordinating Centre (EPPI-Centre).

\section{Subgroup analysis and investigation of heterogeneity}

We will explore potential sources of heterogeneity by carrying out the following subgroup analyses.

- Higher versus lower falls risk at enrolment (i.e. trials with participants selected for inclusion based on history of falling or other specific risk factors for falling, versus trials with unselected participants)

- Individual-versus group-based delivery of the interventions

- Delivery by people with different qualifications or by self (e.g. health professional, trained non-professional, self, or other)

- Duration of the intervention (we will group durations according to whether they are considered brief, standard duration, or long term, relative to the type of intervention being evaluated)

- Mode of content delivery

* Interactive face-to-face e.g. one-to-ones, lectures, workshops, home demonstrations, VR (virtual reality) to retrain how to walk safely etc.

* Interactive virtual/online e.g. mobile phone/smart device apps, webinars, web chats, moderated web forum

* Static virtual/online e.g. web pages, online videos

* Static hard copy e.g. printed leaflets, posters

We will use the test for subgroup differences available in Review Manager Web to determine whether there is evidence for a difference in treatment effect between subgroups.

\section{Sensitivity analysis}

Where possible, we will assess the robustness of our findings by conducting sensitivity analyses. We will examine the effects of the following.

- Removal of studies assessed to be at high risk of bias; and in presence of studies at low risk of bias, we will also assess the impact of removing studies with unclear risk of bias

- The choice of statistical model for pooling (fixed-effect versus random-effects)

- Adjustment for clustering where an intra-cluster correlation coefficient has been borrowed from another study; we will assess the impact of opting for more or less conservative adjustments

- Inclusion of trials which include some participants from institutional care or who had recently returned to the community from hospital 


\section{Assessing the certainty of the evidence and 'Summary of findings' tables}

We will use the GRADE approach to assess the certainty (quality) of the body of evidence for each outcome listed in the 'Types of outcome measures' section (Schunemann 2011). The quality rating 'high' is reserved for a body of evidence based on randomised controlled trials. We may downgrade the quality rating to 'moderate', 'low' or 'very low' depending on the presence and extent of the following five factors: study limitations; inconsistency of effect; imprecision; indirectness; or publication bias. We will prepare a 'Summary of findings' table for each of the main comparisons presented in our review. We will present the primary outcome, rate of falls, and the following secondary outcomes: number of fallers; number of people experiencing one or more fall-related fractures; number of people experiencing one or more falls requiring medical attention; number of people experiencing one or more falls resulting in hospital admission; fall-related psychological outcomes (preferably long-term outcomes; but where these are lacking we will present medium-term outcomes instead); and number of people experiencing one or more adverse events.

\section{ACKNOWLEDGEMENTS}

This review is being supported by the University of Portsmouth, and is not subject to any external funding. We would like to thank our public involvement members for their support in shaping this protocol: Annabelle Glanville-Hearson, Terry Perring, and Sheila Simpson. We have welcomed the support of the Bone, Joint, and Muscle Trauma Cochrane Review Group in the identification of the review question and throughout the review process. We thank Emma Stanmore and Janet Wale for their helpful feedback at external review of the protocol.

This protocol was supported by the National Institute for Health Research (NIHR) via Cochrane Infrastructure funding to the Cochrane Bone, Joint and Muscle Trauma Group. The views and opinions expressed herein are those of the authors and do not necessarily reflect those of the Systematic Reviews Programme, NIHR, National Health Service (NHS) or the Department of Health. 


\section{RE F E R E N C E S}

\section{Additional references}

\section{Adamczewska 2018}

Adamczewska N, Nyman SR. A new approach to fear of falls from connections with the posttraumatic stress disorder literature. Gerontology and Geriatric Medicine 2018;4:doi.org/10.1177/2333721418796238.

\section{Ajzen 1988}

Ajzen I. Attitudes, Personality and Behaviour. 1st Edition. Milton Keynes, UK: Open University Press, 1988.

\section{Alqahtani 2019}

Alqahtani BA, Alshehri MM, Hoover JC, Alenazi AM. Prevalence of falls among older adults in the Gulf Cooperation Council countries: A systematic review and meta-analysis. Archives of Gerontology and Geriatrics 2019;83:169-74. [DOI: 10.1016/ j.archger.2019.04.006]

\section{Aminzadeh 1998}

Aminzadeh F, Edwards N. Exploring seniors' views on the use of assistive devices in fall prevention. Public Health Nursing 1998;15(4):297-304.

\section{Ballinger 2002}

Ballinger C, Payne S. The construction of the risk of falling among and by older people. Ageing and Society 2002;22(3):305-24

\section{Cameron 2018}

Cameron ID, Dyer SM, Panagoda CE, Murray GR, Hill KD, Cumming RG, et al. Interventions for preventing falls in older people in care facilities and hospitals. Cochrane Database of Systematic Reviews 2018, Issue 9. [DOI: 10.1002/14651858.CD005465.pub4]

\section{Canning 2015}

Canning CG, Allen NE, Bloem BR, Keus SHJ, Munneke M, Nieuwboer $A$, et al. Interventions for preventing falls in Parkinson's disease. Cochrane Database of Systematic Reviews 2015, Issue 3. [DOI: 10.1002/14651858.CD011574]

\section{CDHAC 2001}

Commonwealth Department of Health and Aged Care. National falls prevention for older people initiative - Step out with confidence. Canberra: Commonwealth of Australia, 2001.

\section{Chang 2015}

Chang VC, Do MT. Risk factors for falls among seniors: Implications of gender. American Journal of Epidemiology 2015;181(7):521-31. [DOI: 10.1093/aje/kwu268]

\section{Clemson 2004}

Clemson L, Cumming RG, Kendig H, Swann M, Heard R, Taylor K. The effectiveness of a community-based program for reducing the incidence of falls in the elderly: a randomized trial. Journal of the American Geriatrics Society 2004;52(9):1487-94.

\section{Clemson 2019}

Clemson L, Stark S, Pighills AC, Torgerson DJ, Sherrington C, Lamb SE. Environmental interventions for preventing falls in older people living in the community. Cochrane Database of Systematic Reviews 2019, Issue 2. [DOI: 10.1002/14651858.CD013258]

\section{Cost Converter [Computer program]}

CCEMG, EPPI Centre. CCEMG - EPPI-Centre Cost Converter. Version 1.6. London: Evidence for Policy and Practice Information and Coordinating Centre, 2019.

\section{Cumming 1999}

Cumming RG, Thomas M, Szonyi G, Salkeld G, O'Neill E, Westbury C, et al. Home visits by an occupational therapist for assessment and modification of environmental hazards: a randomized trial of falls prevention. Journal of the American Geriatrics Society 1999;47(12):1397-402.

\section{Drahota 2019}

Drahota A, Keenan B, Lachance C, Okunribido O, Bell M, Raftery J. The SAFEST Review: The Shock-Absorbing Flooring Effectiveness SysTematic Review including older adults and staff in care settings. HTA researcher-led evidence synthesis. Available at www.journalslibrary.nihr.ac.uk/programmes/ hta/1714811/\#/.

\section{Drummond 1996}

Drummond MF, Jefferson TO. Guidelines for authors and peer reviewers of economic submissions to the BMJ. The BMJ Economic Evaluation Working Party. BMJ 1996;313(7052):275-83.

\section{Dryden 2012}

Dryden W. Adapting CBT to a broad clientele. In: Dryden W, Branch R editor(s). The CBT Handbook. London: Sage Publications, 2012:214-30.

\section{Duggan 2015}

Duggan M, Ellison NB, Lampe C, Lenhart A, Madden M. Demographics of key social networking platforms. Available at www.pewinternet.org/2015/01/09/demographics-of-key-socialnetworking-platforms-2.

\section{Frith 2017}

Frith J. Fall prevention: empowering people through online education. Annals of Family Medicine 2017;15(5):482.

\section{Garrison 2010}

Garrison KR, Shemilt I, Donell S, Ryder JJ, Mugford M, Harvey I, et al. Bone morphogenetic protein (BMP) for fracture healing in adults. Cochrane Database of Systematic Reviews 2010, Issue 6. [DOI: 10.1002/14651858.CD006950.pub2]

\section{Gill 2000}

Gill TM, Williams CS, Tinetti ME. Environmental hazards and the risk of nonsyncopal falls in the homes of the community-living older person. Medical Care 2000;38(12):1174-83. 


\section{Gillespie 2012}

Gillespie LD, Robertson MC, Gillespie WJ, Sherrington C, Gates S, Clemson LM, et al. Interventions for preventing falls in older people living in the community. Cochrane Database of Systematic Reviews 2012, Issue 9. [DOI: 10.1002/14651858.CD007146.pub3]

\section{Hannan 2010}

Hannan MT, Gagnon MM, Aneja J, Jones RN, Cupples LA, Lipsitz LA, et al. Optimizing the tracking of falls in studies of older participants: comparison of quarterly telephonerecall with monthly falls calendars in the MOBILIZE Boston Study. American Journal of Epidemiology 2010;171(9):1031-6.

\section{Hauer 2006}

Hauer K, Lamb S, Jorstad E, Todd C, Becker C. Systematic review of definitions and methods of measuring falls in randomised controlled fall prevention trials. Age and Ageing 2006;35(1):5-10.

\section{Healey 2007}

Healey F, Scobie S, Glampson B, Pryce A, Joule N, Willmott M. Slips, trips and falls in hospital. The third report from the Patient Safety Observatory. London (UK): National Patient Safety Agency; 2007.

\section{Herdman 2011}

Herdman M, Gudex C, Lloyd A, Janssen M, Kind P, Parkin D, et al. Development and preliminary testing of the new fivelevel version of EQ-5D (EQ-5D-5L). Quality of Life Research 2011;20(10):1727-36.

\section{Higgins 2011}

Higgins JP, Green S, editor(s). Cochrane Handbook for Systematic Reviews of Interventions Version 5.1.0 (updated March 2011). The Cochrane Collaboration, 2011. Available from handbook.cochrane.org.

\section{Higgins 2017}

Higgins JPT, Altman DG, Sterne JAC (editors). Chapter 8: Assessing risk of bias in included studies. In: Higgins JPT, Churchill R, Chandler J, Cumpston MS (editors), Cochrane Handbook for Systematic Reviews of Interventions version 5.2.0 (updated June 2017). www.training.cochrane.org/handbook 2017.

\section{Hopewell 2018}

Hopewell S, Adedire O, Copsey BJ, Boniface GJ, Sherrington C, Clemson L, et al. Multifactorial and multiple component interventions for preventing falls in older people living in the community. Cochrane Database of Systematic Reviews 2018, Issue 7. [DOI: 10.1002/14651858.CD012221.pub2]

\section{Huang 2011}

Huang TT, Yang LH, Liu CY. Reducing the fear of falling among community-dwelling elderly adults through cognitivebehavioural strategies and intense Tai Chi exercise: a randomized controlled trial. Journal of Advanced Nursing 2011;67(5):961-71.

\section{Lamb 2005}

Lamb SE, Jørstad-Stein EC, Hauer K, Becker C, Prevention of Falls Network Europe and Outcomes Consensus Group. Development of a common outcome data set for fall injury prevention trials: the prevention of falls network Europe consensus. Journal of the American Geriatrics Society 2005;53(9):1618-22.

\section{Lamb 2011}

Lamb SE, Becker C, Gillespie LD, Smith JL, Finnegan S, Potter R, et al. Reporting of complex interventions in clinical trials: development of a taxonomy to classify and describe fallprevention interventions. Trials 2011;12:125.

\section{Le 2015}

Le QT, Pedro A, Park CS. A social virtual reality based construction safety education system for experiential learning. Journal of Intelligent and Robotic Systems 2015;79(3-4):487-506. [DOI: 10.1007/s10846-014-0112-z]

\section{Lefebvre 2011}

Lefebvre C, Manheimer E, Glanville J. Chapter 6: Searching for studies. In: Higgins JPT, Green S editor(s). Cochrane Handbook for Systematic Reviews of Interventions Version 5.1.0 (updated March 2011). The Cochrane Collaboration, 2011. Available from handbook.cochrane.org.

Lord 2007

Lord SR, Sherrington C, Menz HB, Close JC. Falls in Older People: Risk Factors and Strategies for Prevention. 1st Edition. Cambridge: Cambridge University Press, 2007.

\section{Mansell 2012}

Mansell W, Taylor JL. What is and what isnt CBT?. In: Dryden W, Branch, R editor(s). The CBT Handbook. London: Sage Publications, 2012:5-24.

\section{NHS 2019}

NHS. Cognitive behavioural therapy (CBT). www.nhs.uk/ conditions/cognitive-behavioural-therapy-cbt (accessed 13 November 2019)..

\section{NICE 2013}

National Institute for Health and Care Excellence (NICE). Falls in older people: assessing risk and prevention: clinical guideline (CG161). Available at www.nice.org.uk/guidance/cg161/chapter/ Introduction.

\section{Nyman 2009}

Nyman SR, Yardley L. Usability and acceptability of a website that provides tailored advice on falls prevention activities for older people. Health Informatics Journal 2009;15(1):27-39.

\section{Nyman 2011}

Nyman S. Psychosocial issues in engaging older people with physical activity interventions for the prevention of falls. Canadian Journal on Aging / La Revue Canadienne du Vieillissement 2011;30(1):45-55. 


\section{O'Shea 2017}

O'Shea L, Watkins E, Farrand P. Psychological interventions for the treatment of depression, anxiety, alcohol misuse or anger in armed forces veterans and their families: systematic review and meta-analysis protocol. Systematic Reviews 2017;6(1):112.

\section{Philips 2006}

Philips Z, Bojke L, Sculpher M, Claxton K, Golder S. Good practice guidelines for decision-analytic modelling in health technology assessment: A review and consolidation of quality assessment. PharmacoEconomics 2006;24(4):355-71. [DOI: doi:10.2165/00019053-200624040-00006]

\section{ProFaNE 2007}

ProFaNE. ProFaNE Taxonomy Manual: Manual for the fall prevention classification system. www.profane.eu.org/ documents/Falls_Taxonomy.pdf (accessed 05 July 2019).

\section{Reventlow 2007}

Reventlow SD. Perceived risk of osteoporosis: restricted physical activities? Qualitative interview study with women in their sixties. Scandinavian Journal of Primary Health Care 2007;25(3):160-5.

\section{Review Manager Web [Computer program]}

The Cochrane Collaboration. Review Mananager Web (RevMan Web). The Cochrane Collaboration, 2019.

\section{Rubenstein 2006}

Rubenstein LZ. Falls in older people: epidemiology, risk factors and strategies for prevention. Age and Ageing 2006;35(Suppl 2):ii37.

\section{Schunemann 2011}

Schünemann HJ, Oxman AD, Higgins JPT, Vist GE, Glasziou P, Guyatt GH. Chapter 11: Presenting results and 'Summary of findings' tables. In: Higgins JPT, Green S editor(s). Cochrane Handbook for Systematic Reviews of Interventions Version 5.1.0 (updated March 2011). The Cochrane Collaboration, 2011. Available from handbook.cochrane.org.

\section{Sherrington 2019}

Sherrington C, Fairhall NJ, Wallbank GK, Tiedemann A, Michaleff ZA, Howard K, et al. Exercise for preventing falls in older people living in the community. Cochrane Database of Systematic Reviews 2019, Issue 1. [DOI: 10.1002/14651858.CD012424.pub2]

\section{Smeeth 2002}

Smeeth L, Ng ESW. Intraclass correlation coefficients for cluster randomized trials in primary care: data from the MRC Trial of the Assessment and Management of Older People in the Community. Controlled Clinical Trials 2002;23(4):409-21.

\section{Speechley 2011}

Speechley M. Unintentional falls in older adults: a methodological historical review. Canadian Journal on Aging / La Revue Canadienne du Vieillissement 2011;30(1):21-32.

\section{Tideiksaar 2002}

Tideiksaar R. Falls in Older People: Prevention \& Management. United Kingdom: Cambridge University Press, 2002.

\section{Tinetti 2010}

Tinetti ME, Kumar C. The patient who falls: "It's always a tradeoff.". JAMA: The Journal of the American Medical Association 2010;303(3):258-66.

\section{Todd 2004}

Todd C, Skelton D. What are the main risk factors for falls among older people and what are the most effective interventions to prevent these falls?. WHO Regional Office for Europe (Health Evidence Network report) 2004.

\section{Verheyden 2013}

Verheyden GS, Weerdesteyn V, Pickering RM, Kunkel D, Lennon S, Geurts ACH, et al. Interventions for preventing falls in people after stroke. Cochrane Database of Systematic Reviews 2013, Issue 5. [DOI: 10.1002/14651858.CD008728.pub2]

\section{WHO 2007}

World Health Organization. WHO Global Report on Falls Prevention in Older Age. France: WHO Press, 2007. [ISBN 97892 41563536 ]

\section{WHO 2018}

World Health Organisation (WHO). Ageing and Health. www.who.int/news-room/fact-sheets/detail/ageing-and-health (accessed 8 July 2019).

\section{WHO 2018a}

World Health Organisation (WHO). Falls: Key Facts. www.who.int/news-room/fact-sheets/detail/falls (accessed 8 July 2019).

\section{Yardley 2002}

Yardley L, Smith H. A prospective study of the relationship between feared consequences of falling and avoidance of activity in community-living older people. Gerontologist 2002;42(1):17-23.

\section{Yardley 2005}

Yardley L, Beyer N, Hauer K, Kempen G, Piot-Ziegler C, Todd C. Development and initial validation of the Falls Efficacy ScaleInternational (FES-I). Age \& Ageing 2005;34(6):614-19.

\section{Yardley 2007}

Yardley L, Nyman SR. Internet provision of tailored advice on falls prevention activities for older people: a randomized controlled evaluation. Health Promotion International 2007;22(2):122-8.

\section{Zijlstra 2007}

Zijlstra GA, van Haastregt JC, van Eijk JT, van Rossum E, Stalenhoef PA, Kempen GI. Prevalence and correlates of fear of falling, and associated avoidance of activity in the general population of community-living older people. Age and Ageing 2007;36(3):304-9. 


\section{A P P E N D C E S}

\section{Appendix 1. Example search strategy (for MEDLINE via Ovid)}

\section{Accidental Falls/}

2 (falls or faller ${ }^{\star} 1$ ).tw.

3 or/ $1-2$

4 exp Aged/

5 (senior ${ }^{\star} 1$ or elder ${ }^{\star}$ or old* or aged or ag?ing or postmenopausal or community dwelling).tw.

6 or $/ 4-5$

73 and 6

8 Randomized controlled trial.pt.

9 Controlled clinical trial.pt.

10 randomized.ab.

11 placebo.ab.

12 Clinical trials as topic/

13 randomly.ab.

14 trial.ti.

158 or 9 or 10 or 11 or 12 or 13 or 14

16 exp Animals/ not Humans/

1715 not 16

187 and 17

$19\left(201203^{\star}\right.$ or $201204^{\star}$ or $201205^{\star}$ or $201206^{\star}$ or $201207^{\star}$ or $201208^{\star}$ or $201209^{\star}$ or $201210^{\star}$ or $201211^{\star}$ or $201212^{\star}$ or $2013^{\star}$ or $2014^{\star}$ or $2015^{\star}$ or $2016^{\star}$ or $2017^{\star}$ or $2018^{\star}$ or $\left.2019^{\star}\right)$.ed,dc.

2018 and 19

\section{Appendix 2. 'Risk of bias' assessment tool*}

\section{Domain Criteria for judging risk of bias}

\section{Random sequence genera-}

tion relating to selection bias (biased allocation to interventions) due to inadequate generation of a randomised sequence
- Judgement of 'low risk' if the trial authors described a random component in the sequence generation, e.g. referring to a random number table; using a computer random number generator; coin tossing; shuffling cards or envelopes; throwing dice; drawing of lots; minimisation.

- Judgement of 'high risk' if the trial used a systematic non-random method, e.g. date of admission; odd or even date of birth; case record number; clinician judgement; participant preference; patient risk factor score or test results; availability of intervention.

- Judgement of 'unclear risk' if there is insufficient information about the sequence generation process to permit judgement of 'low risk' or 'high risk'.

\section{Allocation concealment relat- ing to selection bias (biased al- location to interventions) due to inadequate concealment of allocations prior to assign- ment}

- Judgement of 'low risk' in studies using:

* individual randomisation if the trial described allocation concealment as by central allocation (telephone, internet-based, or pharmacy-controlled randomisation); sequentially numbered, identical drug containers; sequentially numbered, opaque, sealed envelopes;

* cluster randomisation if allocation of all cluster units performed at the start of the study and individual participant recruitment was completed prior to assignment of the cluster, and the same participants were followed up over time or individual participants were recruited after cluster assignment, but recruitment was carried out by a person unaware of group allocation and participant characteristics (e.g. fall history), or individual participants in intervention and control arms were invited by mail questionnaire with identical information.

- Judgement of 'high risk' in studies using:

* individual randomisation if investigators enrolling participants could possibly have foreseen assignments thus introducing selection bias, e.g. using an open random allocation schedule (e.g. a list of random numbers); assignment envelopes unsealed, non-opaque, or not sequentially numbered; alternation or rotation; date of birth; case record number; or any other explicitly unconcealed procedure;

* cluster randomisation if individual participant recruitment was undertaken after group allocation by a person who was unblinded and may have had knowledge of participant characteristics. 
- Judgement of 'unclear risk' if insufficient information to permit judgement of 'low risk' or 'high risk'. This is usually the case if the method of concealment is not described or not described in sufficient detail to allow a definitive judgement, e.g. if the use of assignment envelopes is described, but it is unclear whether envelopes were sequentially numbered, opaque, and sealed.

Blinding of participants and personnel relating to performance bias due to knowledge of the allocated interventions by participants and personnel carrying out the interventions
- Judgement of 'low risk' if blinding of participants and personnel implementing the interventions was ensured and it is unlikely that the blinding could have been broken, or blinding was not done but the review authors judge that the outcomes (falls and fractures) are unlikely to be influenced by lack of blinding.

- Judgement of 'high risk' if participants or intervention delivery personnel, or both were not blinded to group allocation (e.g. exercise intervention), and the outcomes (falls and fractures) are likely to be influenced by lack of blinding.

- Judgement of 'unclear risk' if there is insufficient information to permit a judgement of 'low risk' or 'high risk'.
Blinding of outcome assessment relating to detection bias due to knowledge of the allocated interventions by outcome assessors
- Falls, fallers:

* judgement of 'low risk' if outcomes were recorded/confirmed in all allocated groups using the same method and the personnel recording/confirming outcomes were blind to group allocation;

* judgement of 'high risk' if outcomes were not recorded/confirmed in all allocated groups using the same method or the personnel recording/confirming outcomes were NOT blind to group allocation;

* judgement of 'unclear' if there is insufficient information to permit a judgement of 'low risk' or 'high risk'.

- Fractures:

* judgement of 'low risk' if fractures were recorded/confirmed in all allocated groups using the same method, and fractures were confirmed by the results of radiological examination or from primary care case records and the personnel recording/confirming fractures were blind to group allocation;

* judgement of 'high risk' if fractures were not recorded/confirmed in all allocated groups using the same method, or the only evidence for fractures was from self-reports from participants or carers;

* judgement of 'unclear risk' if there is insufficient information to permit a judgement of 'low risk' or 'high risk'.
Incomplete outcome data relating to attrition bias due to amount, nature, or handling of incomplete outcome data
- Judgement of 'low risk' if there are no missing outcome data, or less than $20 \%$ of outcome data are missing and losses are balanced in numbers across intervention groups with similar reasons for missing data across groups, or missing data have been imputed using appropriate methods.

- Judgement of 'high risk' if greater than $20 \%$ of outcome data are missing, or reasons for missing outcome data are likely to be related to true outcome, with either imbalance in numbers or reasons for missing data across intervention groups, or 'as-treated' analysis done with substantial departure of the intervention received from that assigned at randomisation or potentially inappropriate application of simple imputation.

- Judgement of 'unclear risk' if there is insufficient information to permit a judgement of 'low risk' or 'high risk'.
Selective outcome reporting relating to bias due to the selective reporting or non-reporting of findings
- Judgement of 'low risk' if the study protocol is available and all prespecified study outcomes are reported in the prespecified way, or the study protocol is unavailable but it is clear that the published report includes all expected outcomes.

- Judgement of 'high risk' if not all prespecified study outcomes are reported, or one or more primary outcomes are reported in ways that were not prespecified, or one or more outcomes are reported incompletely or the study fails to include results for a key outcome that would be expected to have been reported.

- Judgement of 'unclear risk' if there is insufficient information to permit a judgement of 'low risk' or 'high risk'. 
(Continued)

Method of ascertaining falls relating to bias in the recall of falls due to unreliable methods of ascertainment
- Judgement of 'low risk' if the study used some form of concurrent collection of data about falling, e.g. participants were given postcards to fill in daily and mail back monthly, calendar to mark monthly, or, more frequently, follow-up by the researchers.

- Judgement of 'high risk' if ascertainment relied on participant recall at longer intervals than one month during the study or at its conclusion.

- Judgement of 'unclear risk' if there was retrospective recall over a short period only, or if the trial authors did not describe details of ascertainment, i.e. insufficient information was provided to permit a judgement of 'low risk' or 'high risk'.

\section{Cluster-randomised trials} relating to bias due to factors particular to cluster-randomised trials
- Judgement of 'low risk' if the study predominantly had the following characteristics:

* individuals were recruited to the trial prior to randomisation of the clusters;

* baseline comparability of clusters was reported or there was statistical adjustment for baseline characteristics;

* no loss of clusters or missing outcomes for individuals within specific clusters;

* clustering is accounted for in the analyses;

* results are comparable with individually randomised trials.

- Judgement of 'high risk' if the study predominantly had the following characteristics:

* individuals were recruited to the trial after randomisation of the clusters;

* baseline comparability of clusters was not reported, and there was no statistical adjustment for baseline characteristics;

* loss of entire clusters or missing outcomes for individuals within clusters;

* no accounting for clustering in analyses;

* results not comparable with individually randomised trials.

- Judgement of 'unclear risk' if there is insufficient information to permit a judgement of 'low risk' or 'high risk'.

*This was adapted from Table 8.5.a 'The Cochrane Collaboration's tool for assessing risk of bias' and Table 8.5.d 'Criteria for judging risk of bias in the 'Risk of bias' assessment tool' (Higgins 2011) by authors of falls reviews, initially Hopewell 2018.

\section{CONTRIBUTIONSOFAUTHORS}

JU, AD and HM all contributed to writing the protocol.

$\mathrm{JU}$ is the guarantor of the protocol and will act as guarantor of the review.

\section{Contributions of the editorial base}

Helen Handoll (Co-ordinating Editor): edited the protocol; advised on methodology and protocol content; and approved the final version for publication.

Joanne Elliott (Managing Editor): coordinated the editorial process; advised on content; and edited the protocol.

Maria Clarke (Information Specialist): advised on the search strategy and edited the Search methods for identification of studies section.

\section{DECLARATIONSOF INTEREST}

JU has no known conflicts of interest.

HM has no known conflicts of interest.

AD is Principal Investigator for another systematic review exploring the use of shock-absorbing flooring in hospitals and care homes for fall-related injury prevention, funded by National Institute for Health Research (NIHR Health Technology Assessment, 17/148/11 - The SAFEST Review: The Shock-Absorbing Flooring Effectiveness SysTematic Review including older adults and staff in care settings).

\section{SOURCES OF SUPPORT}

\section{Internal sources}

- University of Portsmouth, UK. 


\section{External sources}

- No sources of support supplied 